\title{
Mesoscopic Models of Neurotransmission as Intermediates between Disease Simulators and Tools for Discovering Design Principles
}

\section{E. O. Voit, Z. Qi, S. Kikuchi}

Pharmacopsychiatry 2012; Suppl. 1: S22-S30

DOI: $10.1055 / s-0032-1304653$

This article was published with a misspellt authors name. Z. Qui was changed to Z. Qi. 